\title{
The Global Formulation of the Cauchy Problem
}

\author{
Mohammad Ali Bashir ${ }^{1,2}$ \& Tarig Abdelazeem Abdelhaleem ${ }^{3}$ \\ ${ }^{1}$ Department of Mathematics, Faculty of Sciences, University of Alnillin, Khartoum, Sudan. \\ ${ }^{2}$ Academy of Engineering Sciences, Khartoum, Sudan. \\ ${ }^{3}$ Department of Mathematics, collage of Applied and Industrial Science, University of Bahri, Khartoum, Sudan \\ Correspondence: Marina Victorovna Yashina, E-mail: yash-marina@yandex.ru
}

Received: March 7, 2016 Accepted: March 25, 2016 Online Published: May 18, 2016

doi:10.5539/jmr.v8n3p74 URL: http://dx.doi.org/10.5539/jmr.v8n3p74

\begin{abstract}
A Geometrical model for the global Cauchy problem, generalizing the traditional Cauchy problem is considered .The complete correspondence between the known analytical formulation and the geometrical interpretation is described, we have utilized the generalized Green's function and the open mapping theorem appropriate to the problem.
\end{abstract}

Keywords: Cauchy problem, Green's function, Globally Hyperbolic space time, Open mapping theorem, semi-Riemannian metric.

\section{Introduction}

In this paper we discuss the global formulation of the Cauchy problem(Bar, Ginoux, \& Pfaffe, 2007; Minguzzi \& Sánchez, 2008), and its solution for globally hyperbolic space time(Beem, Ehrlich, \& Easley, 1996; O'neill, 1983). Also we discuss the role of open mapping theorem(Bär \& Ginoux, 2012; Kreyszig, 1989), in our solution, because of its various properties. The open mapping theorem seems to be a good tool for investigating that for general maps between topological spaces. For the formulation of the global Cauchy problem we need to know two kinds of structure, the first is a time orientation which separates future from past(Bär \& Fredenhagen, 2009), the second ingredient is that of a hyper surface $\Sigma$ in which we can specify the initial values. In order to approach the global existence of solutions we assume that $M$ is globally hyperbolic with a smooth spacelike Cauchy hyper surface $\Sigma$. For every $p \in M$ we have a unique time $t$ with $p \in \Sigma_{t}$, on each $\Sigma_{t}$ (Mühlhoff, 2011), we also have a Riemannian metric $g_{t}$ such that $g=\beta d t^{2}-g_{t}$,

\section{Preliminaries}

2.1 Cauchy Problem in the (n-1)-dimensional Subspace $E_{n-1}$ (Stakgold \& Holst, 2011),

Let $\mathrm{G}$ be a domain in the (n-1)-dimensional subspace $E_{n-1}$ of the variable, $x_{1}, x_{2}, \ldots ., x_{n-1}$. then the following is a Cauchy problem :

$$
\sum_{i=1}^{n-1} \frac{\partial^{2} u}{\partial x_{i}^{2}}-\frac{\partial^{2} u}{\partial x_{n}^{2}}=0
$$

Satisfying the conditions

$$
\begin{aligned}
& u\left(x_{1}, x_{2}, \ldots, x_{n-1}, 0\right)=f(\bar{x}) \\
& {\left[\frac{\partial u\left(x_{1}, \ldots, x_{n-1}, x_{n}\right)}{\partial x_{n}}\right]_{x_{n}=0}=g(\bar{x})}
\end{aligned}
$$

For $\bar{x}=\left(x_{1}, x_{2}, \ldots, x_{n-1}\right) \in G$ and $\mathrm{f}$, g are sufficiently smooth functions defined in G. Conditions (2),(3) are called Cauchy conditions or initial conditions f, g are called Cauchy data and the system (1), (2) and (3) is called a Cauchy problem, $G$ is called the initial manifold. In the IVP G is the hypersurface obtained by the intersection of the n-dimensional region $\mathrm{T}$ and the hyperplane $x_{n}=0$. An initial domain may not be a proper subset of the boundary, for example in $E_{2}$ consisting of point $(\mathrm{x}, \mathrm{t})$, the initial domain may be $\boldsymbol{t}=\mathbf{O}$ or a subset of it. In general elliptic equations are associated with boundary conditions and hyperbolic and parabolic equations with initial conditions. 
2.2 (Example) : take the PDE

$\frac{\partial^{2} \boldsymbol{u}}{\partial \boldsymbol{x}^{2}}-\frac{\partial^{2} \boldsymbol{u}}{\partial \boldsymbol{t}^{2}}=\mathbf{O}$ with ICs : $u(x, 0)=f(x), \frac{\partial u}{\partial t}=g(x)$

The D' Alembert's solution to the Cauchy problem is

$$
u(x, t)=\frac{1}{2} f(x+t)+\frac{1}{2} f(x-t)+\frac{1}{2} \int_{x-t}^{x+t} g(s) d s, t>0
$$

The solution exists, is unique and depends continuously on the data $f(x)$ and $g(x)$. Hence the Cauchy problem for the wave equation is well-posed.

\subsection{Semi-Riemannian Metric}

A section $g \in \Gamma^{\infty}\left(S^{2} T^{*} M\right)$ is called semi-Riemannian metric if the bilinear form $g_{p} \in S^{2} T_{p}{ }^{*} M$ on $T_{p} M$ is non-degenerate for all $p \in M$. If in addition $g_{p}$ is positive definite for all $p \in M$ then $g$ is called Riemannian metric. If $g_{p}$ has signature $(+,-, \ldots,-)$ then $g$ is called Lorentz metric.

\subsection{Causal Subsets}

Let $U \subseteq M$ be an open subset. Then $U$ is called causal if there is a geodesically convex open subset $U^{\prime} \subseteq M$ such that $U^{c 1} \subseteq U^{\prime}$ and for any two points $p, q \in U^{c 1}$, the diamond $J_{U^{\prime}}(p, q)$ is compact and contained in $U^{c 1}$.

\subsection{A Causal and Achronal Subsets}

Let $A \subseteq M$ be a subset of a time-oriented Lorentz manifold. Then $A$ is called

i.) a chronal if every timelike curve intersects $A$ in at most one point.

ii.) a causal if every causal curve intersects $A$ in at most one point

\section{6 (Theorem) A Chronal Hyper Surfaces}

Let $(M, g)$ be a time-oriented Lorentz manifold and $A \subseteq M$ a chronal. Then $A$ is a topological hyper surface in $M$ if and only if $A$ does not contain any of its edge points.

\subsection{Cauchy Hyper Surface}

Let $(M, g)$ be a time-oriented Lorentz manifold. A subset $\Sigma \subseteq M$ is called a Cauchy hyper surface if every inextensible timelike curve meets $\Sigma$ in exactly one point.

\subsection{Cauchy development}

Let $A \subseteq M$ be a subset. The future Cauchy development $D_{M}^{+}(A) \subseteq M$ of $A$ is the set of all those points $p \in M$ for which every past-inextensible causal curve through $p$ also meets $A$ Alogously, one defines the past Cauchy development $D_{M}^{-}(A)$ and we call

the Cauchy development of $A$.

$$
D_{M}(A)=D_{M}^{+}(A) \cap D_{M}^{-}(A)
$$

\subsection{Globally Hyperbolic Spacetime}

A time-oriented Lorentz manifold $(M, g)$ is called globally hyperbolic if

i.) $(M, g)$ is causal,

ii.) all diamonds $J_{M}(p, q)$ are compact for $p, q \in M$.

\subsection{Time Function(Baer \& Strohmaier, 2015)}

Let $(M, g)$ be a time-oriented Lorentz manifold and $t: M \rightarrow R$ a continuous function. Then $t$ is called a

i.) time function if $t$ is strictly increasing along all future directed causal curves.

ii.) temporal function if $t$ is smooth and grad $t$ is future directed and timelike.

i.) Cauchy time function if $t$ is a time function whose level sets are Cauchy hypersurfaces.

v.) Cauchy temporal function if $t$ is a temporal function such that all level sets are Cauchy hyper surfaces. 
2.11. Theorem(Baer \& Strohmaier, 2015),

Let $(M, g)$ be a connected time-oriented Lorentz manifold. Then the following statements are equivalent:

i.) $(M, g)$ is globally hyperbolic.

ii.) There exists a topological Cauchy hypersurface.

iii.) There exists a smooth spacelike Cauchy hypersurface.

In this case there even exists a Cauchy temporal function $t$ and $(M, g)$ is isometrically diffeomorphic to the product manifold

$$
R \times \Sigma \text { with metric } g=\beta d t^{2}-g t,
$$

where $\beta \in \ell^{\infty}(R \times \Sigma)$ is positive and $g_{t} \in \Gamma^{\infty}\left(S^{2} T^{*} \Sigma\right)$ is a Riemannian metric on $\Sigma$ depending smoothly on $t$. Moreover, each level set

$$
\Sigma_{t}=\{(t, \sigma) \in R \times \Sigma\} \subseteq M
$$

of the temporal function $t$ is a smooth spacelike Cauchy hypersurface.

\section{Existence of Global Solutions to the Cauchy Problem}

\subsection{Proposition}

Let $(M, g)$ be a time-oriented Lorentz manifold with a smooth spacelike hyper surface $\imath: \sum \mapsto M$ with future directed normal vector field $n$. Moreover, let $U \subseteq U^{c 1} \subseteq U^{\prime}$ be a sufficiently small causal open subset of M such that $\Sigma \cap U \mapsto U$ is a Cauchy hyper surface for $U$. Then there exists a unique solution $u \in \Gamma^{\infty}\left(\left.E\right|_{U}\right)$ for given initial values $u_{0}, \dot{u}_{0} \in \Gamma_{0}^{\infty}\left(\left.\imath^{\#} E\right|_{U}\right)$ and given inhomogeneity $v \in \Gamma_{0}^{\infty}\left(\left.E\right|_{U}\right)$ of the inhomogeneous wave equation

$$
D u=v
$$

with $\quad \imath^{\#} u=u_{0}$, and $\imath^{\#} \nabla_{n}^{E} u=\dot{u}_{0}$. in addition we have

$$
\operatorname{supp} u \subseteq J_{M}\left(\operatorname{supp} u_{0} \cup \operatorname{supp} \dot{u}_{0} \bigcup \operatorname{supp} v\right)
$$

\subsection{Theorem}

Let $(M, g)$ be a globally hyperbolic and let $\imath: \Sigma \mapsto M$ be a smooth spacelike Cauchy hypersurface with future directed normal vector field $n \in \Gamma^{\infty}\left(\left.l^{\#} E\right|_{U}\right)$. Assume that is a solution to the wave equation $D u=0$ with initial conditions

$$
u_{0}=0=\dot{u}_{0}
$$

then

$$
u=0
$$

Moreover to develop our constructing we assume that $\mathrm{M}$ is globally hyperbolic with $\Sigma$ is smooth spacelike. For every $p \in \mathrm{M}$ we have a unique time $t$ with $p \in \Sigma_{t}$. we have a Riemannian metric $g_{t}$ such that $g=\beta d t^{2}-g_{t}$ on each $\Sigma_{t}$.and open Ball $B_{r}(p)_{t}$ such $B_{r}(p) \subseteq \Sigma_{t}$ is open $n \Sigma_{t}$ but not in $\mathrm{M}$.Then

$$
d_{g t}(p, q)=\inf \left\{\int_{a}^{b} g t(\dot{\gamma}(\tau), \dot{\gamma}(\tau)) d \tau \mid \gamma(a)=p, \gamma(b)=q, \gamma(\tau) \in \Sigma_{t}\right\}
$$

where $\gamma$ is an at least piecewise $\ell^{1}$ curve joining $p, q \in \Sigma_{t}$ inside $\Sigma_{t}$.consider its Cauchy development $D_{M}\left(B_{r}(p)\right)=D_{M}^{+}((p)) \cup D_{M}^{-}\left(B_{r}(p)\right)$ in $M$ according to Definition.Now we want to find $r$ small enough that $D_{M}\left(B_{r}(p)\right)$ is a nice open neighbourhood of $p$ allowing a local fundamental solution[4].

\subsection{Lemma,(Waldmann, 2012)}

The function $\rho: M \rightarrow(0,+\infty]$ defined by

$$
\rho(p)=\sup \left\{r>0 \mid D\left(B_{r}(p)\right) i s R C C S V\right\}
$$

Is well defined and lower semi-continuous.

\subsection{Lemma}

for every point $p \in \mathrm{M}$ and $r>0$ the exists a $t>0$ such that

$$
J_{m}\left(B_{\frac{r}{2}}(p)^{c 1}\right) \cap([t-\tau, t+\tau] \times \Sigma) \subseteq D_{M}\left(B_{r}(p)\right)
$$

Where $t \in R$ is the uniqe time with $p \in \Sigma_{t}$. 


\subsection{Lemma}

The function $\theta_{r}: \mathrm{M} \rightarrow(0, \infty)$ is well-defined and lower semi-continuous, where

$$
\theta_{r}=\sup \left\{\tau>0 \mid J_{m}\left(B_{\frac{r}{2}}(p)^{c 1}\right) \cap([t-\tau, t+\tau] \times \Sigma) \subseteq D_{M}\left(B_{r}(p)\right)\right\}
$$

\subsection{Lemma (Waldmann, 2012)}

let $K \subseteq \mathrm{M}$ be compact then there is a $\delta>0$ such that for all times $t \in R$ and all $u_{t}, \dot{u}_{t} \in \Gamma^{\infty}\left(l_{t}{ }^{*} E\right)$ on $\sum_{t}$ with support Supp $u_{t}$, Supp $\dot{u}_{t} \subseteq K$, We have smooth solution $u$ of the homogeneous Wave Equation $D u=0$, on the time slice $(t-\delta, t+\delta) \times \Sigma$, with the initial conditions $\left.u\right|_{\Sigma_{t}}=u_{t}$ and $\left.\nabla_{n}^{E} u\right|_{\Sigma_{t}}=\dot{u}_{t}$ Moreover for the support we have

$$
\text { Supp } u \subseteq J_{M}\left(\operatorname{Supp} u_{t} \cup \operatorname{Supp} \dot{u}_{t}\right)
$$

Proof:

Since $\rho$ is lower semi-continous according to lemma 2-1 and positive, it admits a minimum on the compact subset $K$. Thus we find $r>0$ with $\rho(p)>2 r_{0}$ for all $p \in k$ so for this radius the function $\theta_{2 r 0}$ is lower semi-continuous according lemma 3.3 and positive. And we can find $\delta>0$ with $\theta_{2 r 0}>\delta$ on $K$, given $t \in R, \Sigma_{t} \cap \mathrm{K}$ is compact. We can cover it with open balls $B_{r 0}\left(p_{1}\right), \ldots \ldots, B_{r 0}\left(p_{N}\right)$ of radius $r_{0}$. Also we can find $\chi_{1}, \ldots ., \chi_{n}$ subordinate to $B_{r 0}\left(p_{1}\right) \cup, \ldots \ldots ., \cup B_{r 0}\left(p_{N}\right)$

Then we have $\chi_{1}, \ldots, \chi_{N}=1$, and Supp $\chi_{\alpha} \subseteq B_{r 0}\left(p_{\alpha}\right)$ for all $\alpha=1, \ldots . N$. let $u_{t}$ and $\dot{u}_{t}$ in $B_{r 0}\left(p_{\alpha}\right)$ by considering $\chi_{\alpha} u_{t}, \chi_{\alpha} \dot{u}_{t}$ respectively ,with $\chi_{\alpha} u_{t}, \chi_{\alpha} \dot{u}_{t} \in \Gamma_{0}^{\infty}\left(u_{t}^{\#} E\right)$, and $\chi_{1} u_{t}+\ldots .+\chi_{N} u_{t}=u_{t}$ and $\chi_{1} \dot{u}_{t}+\ldots .+\chi_{N} \dot{u}_{t}=\dot{u}_{t}$, Then $D_{M}\left(B_{2 r 0}\left(p_{\alpha}\right)\right)$, is still RCCSV.

Then we can use the proposition 3.1 to obtain smooth solution , $u_{\alpha} \in \Gamma^{\infty}\left(\left.E\right|_{D M\left(B_{2 r_{0}}\left(p_{\alpha}\right)\right)}\right)$ of the homogeneous wave equation $D u_{\alpha}=0$,on $D_{M}\left(B_{2 r 0}\left(p_{\alpha}\right)\right)$, For the initial conditions, $u_{\left.\alpha\right|_{\Sigma_{t}}}=\chi_{\alpha} u_{t}$ and $\left.\nabla_{n}^{E} u_{\alpha}\right|_{\Sigma_{t}}=\chi_{\alpha} \dot{u}_{t} \cdot$ and Then

$$
\text { Supp } u_{\alpha} \subseteq J_{M}\left(\operatorname{Supp} \chi_{\alpha} u_{t} \cup \operatorname{Supp} \chi_{\alpha} \dot{u}_{t}\right)
$$

By the Definition of the function $\theta_{2 r o}$ and the choice of $\delta$ we see that

$$
J_{M}\left(B_{r 0}\left(p_{\alpha}\right)^{c 1} \cap([t-\delta, t+\delta] \times \Sigma) \subseteq D_{M}\left(B_{2 r 0}\left(p_{\alpha}\right)\right),\right.
$$

Since

$u_{\alpha}$ is difined on $J_{M}\left(B_{r 0}\left(p_{\alpha}\right)^{c 1} \cap([t-\delta, t+\delta] \times \Sigma)\right.$, and Supp $\chi_{\alpha} u_{t}$, Supp $\chi_{\alpha} \dot{u}_{t} \subseteq\left(B_{r 0}\left(p_{\alpha}\right)\right.$ from (16) we find

$$
\text { Supp } u_{\alpha} \subseteq J_{M}\left(B_{r 0}\left(p_{\alpha}\right)^{c 1}\right) \text {, }
$$

since $u_{\alpha}$ is smooth on $D_{M}\left(B_{2 r 0}\left(p_{\alpha}\right)\right)$, we can extend $u_{\alpha}$ to $([t-\delta, t+\delta] \times \Sigma)$

Then $\quad u_{\alpha} \in \Gamma^{\infty}\left(\left.E\right|_{([t-\delta, t+\delta] \times \Sigma}\right)$ satisfying $\operatorname{Suppu}_{\alpha} \subseteq J_{M}\left(B_{r 0}\left(p_{\alpha}\right)^{c 1} \cap([t-\delta, t+\delta] \times \Sigma)\right.$, and $D u_{\alpha}=0$ as well as $u_{\left.\alpha\right|_{\Sigma_{t}}}=\chi_{\alpha} u_{t}$ and $\left.\nabla_{n}^{E} u_{\alpha}\right|_{\Sigma_{t}}=\chi_{\alpha} \dot{u}_{t}$.

since $\chi_{\alpha}$ is partition of unity, finally

$$
\begin{gathered}
\text { Supp } u \subseteq \operatorname{Supp} u_{1} \cup \ldots \ldots \cup \operatorname{Supp} u_{N}, \\
\subseteq J_{M}\left(\operatorname{Supp} \chi_{1} u_{t} \cup \operatorname{Supp} \chi_{1} \dot{u}_{t}\right) \cup \ldots \ldots . J_{M}\left(\operatorname{Supp} \chi_{N} u_{t} \cup \operatorname{Supp} \chi_{N} \dot{u}_{t}\right), \\
\subseteq J_{M}\left(\operatorname{Supp} \chi_{1} u_{t} \cup \operatorname{Supp} \chi_{1} \dot{u}_{t} \cup \operatorname{Supp} \chi_{N} u_{t} \cup \operatorname{Supp} \chi_{N} \dot{u}_{t}\right), \\
\subseteq J_{M}\left(\operatorname{Supp} u_{t} \cup \operatorname{Supp} \dot{u}_{t}\right),
\end{gathered}
$$

Since $J_{M}(A) \cup J_{M}(B) \subseteq J_{M}(A \cup B)$ and Supp $\chi_{\alpha} u_{t} \subseteq$ Supp $u_{t}$ and Supp $\chi_{\alpha} \dot{u}_{t} \subseteq$ Supp $\dot{u}_{t}$ for all $\alpha$. this completes the proof.

3.7. Theorem (Waldmann, 2012),

Let $(M, g)$ be a globally hyperbolic spacetime with smooth spacelike Cauchy hyper-surface $\imath: \Sigma \rightarrow M$. 
i.) for $u_{0}, \dot{u}_{0} \in \Gamma_{0}^{\infty}\left(i^{\#} E\right)$ and $v \in \Gamma_{0}^{\infty}(E)$, there exists a unique global solution $u \in \Gamma^{\infty}(E)$ of the inhomogeneous wave equation $D u=v$ with initial conditions, $\imath^{\#} u=u_{0}$ and $\imath^{\#} \nabla_{n}^{E} u=u_{0}$, We have

$$
\operatorname{supp} u \subseteq J_{M}\left(\operatorname{supp} u_{0} \cup \operatorname{supp} \dot{u}_{0} \cup \operatorname{supp} v\right)
$$

ii.) For $k \geq 2$ and $u_{0} \in \Gamma_{0}^{2(k+n+1)+2}\left(i^{\#} E\right), u_{0} \in \Gamma_{0}^{2(k+n+1)+1}\left(i^{\#} E\right)$ and $v \in \Gamma_{0}^{2(k+n+1)}(E)$ there exists a unique global solution $u \in \Gamma^{k}(E)$ of the inhomogeneous wave equation $D u=v$ with initial conditions $\imath^{\sharp} u=u_{0}$ and $\imath^{\sharp} \nabla_{n}^{E} u=\dot{u}_{0} \quad$. It also satisfies (18).

proof

let Supp $u_{0}$,Supp $\dot{u}_{0}$, and Supp $v \subset U \subseteq U^{c 1} \subseteq U^{\prime}(R C C S V) \quad$ and $\quad$ set Supp $u_{0} \cup \operatorname{Supp} \dot{u}_{0} \cup$ and Supp $v \subseteq U$ which is compact then $\mathrm{f}$ we have $k \subseteq(-\in, \in) \times \Sigma$ and $J_{M}(k) \cap((-\in, \in) \times \Sigma) \subseteq U$, for $\in>0$ let $u \in \Gamma^{\infty}\left(\left.E\right|_{U}\right)$ be the solution according to Proposition 3.1.

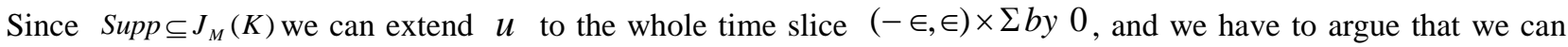
extend this solution to large time slices $(-T, T) \times \Sigma$. we solve $D w=0$ for the initial conditions

$w_{\Sigma_{\Sigma_{t}}}=u_{\Sigma_{\Sigma_{t}}}$ and $\left.\nabla_{n}^{E} w\right|_{\Sigma_{t}}=\left.\nabla_{n}^{E} u\right|_{\Sigma_{t}}$ by using Lemma 2.4 .

Then on $(t-\eta, t+\eta) \times \Sigma, \quad v$ is vanishes by Supp $v \subseteq k$ since $k \subseteq(-\epsilon, t) \times \Sigma$.

and $w, v$ both solve the $D w=0$ with same initial condition on $\Sigma_{t}$,

Then $w=u$ on $(-\in, t) \times \Sigma$ by the uniqueness theorem and shows that $w$ extend $u$ to the slice $(-\in, t+\infty) \times \Sigma$ in smooth way. and $w \subseteq J_{M}(k) \cap \Sigma_{t}$

For the future of $t$ means that Supp wis still contained in $J_{M}(k)$

For the past of $t$ we already know that $w=u$ whence in total $\operatorname{SUPP} w \subseteq J_{M}(k)$.

\section{Global Green Functions and Cauchy Problem}

in this part we, show the Well-posedness of the Cauchy problem with respect to the usual locally convex topologies of smooth or $\ell^{k}-$ sections,

\subsection{Open mapping Theorem}

Let $\varepsilon, \widetilde{\varepsilon}$ be Fréchet spaces and let $\phi: \varepsilon \rightarrow \widetilde{\varepsilon}$ be a continuous linear map. If $\phi$ is surjective then $\phi$ is an open map. As usual, a map $\phi$ is called open if the images of open subsets are again open

\subsection{Corollary}

Let $\phi: \varepsilon \rightarrow \widetilde{\varepsilon}$ be a continuous linear bijection between Fréchet spaces. Then $\phi^{-1}$ is continuous as well. Indeed, let $U \subseteq \mathcal{\varepsilon}$ be open. Then the set-theoretic $\left(\phi^{-1}\right)^{-1}(U)$, i.e. the pre-image of $U$ under $\phi^{-1}$, coincides simply with $\phi(U)$ which is open by the theorem. Thus $\phi^{-1}$ is continuous. Take the result of theorem(2.5).

$$
\Gamma_{0}^{\infty}\left(i^{\#} E\right) \oplus \Gamma_{0}^{\infty}\left(i^{\#} E\right) \oplus \Gamma_{0}^{\infty}(E) \rightarrow \Gamma^{\infty}(E),
$$

Sending $\left(u_{0}, \dot{u}_{0}, v\right)$ to the unique solution $u$ of the Wave Equation $D u=v$ with initial conditions $u_{0}$ and $\dot{u}_{0}$.

\section{3. (Theorem) Well-posed Cauchy Problem}

Let $(M, g)$ be a globally hyperbolic spacetime with smooth spacelike Cauchy hyper surface $t: \Sigma \rightarrow M$. Then the linear map (19) sending the initial conditions and the inhomogeneity to the corresponding solution of the Cauchy problem is continuous.

\section{4. (Theorem) Well-posed Cauchy problem II}

Let $(M, g)$ be a globally hyperbolic spacetime with smooth spacelike Cauchy hyper surface $\imath: \Sigma \rightarrow M$ and let $k \geq 2$. Then the linear map

$$
\Gamma_{0}^{2(k+n+1)+2}\left(\imath^{\#} E\right) \otimes \Gamma_{0}^{2(k+n+1)+1}\left(\iota^{\#} E\right) \otimes \Gamma_{0}^{2(k+n+1)}(E) \rightarrow \Gamma^{k}(E)
$$

sending $\left(u_{0}, u_{0}, v\right)$ to the unique solution $u$ of the inhomogeneous wave equation $D u=v$ with initial $\imath^{\#} u=u_{0}$ and $\imath^{\#} \nabla_{n}^{E} u=\dot{u}_{0} \quad$ continuous.

\section{5. (Theorem )}

Let $(M, g)$ be a globally hyperbolic spacetime and $D \in \operatorname{Diffop}^{2}(E)$ a normally hyperbolic differential operator. For every point $P \in \mathrm{M}$ there is a unique advanced and retarded fundamental solution $F_{M}^{ \pm}(P)$ of Dat $p$. Moreover, for every test section $\varphi \in \Gamma_{0}^{\infty}\left(E^{*}\right)$ the section. 


$$
\mathrm{M} э P \mapsto F_{M}^{ \pm}(P) \varphi \in E_{p}^{ \pm}
$$

is a smooth section of $E^{*}$ which satisfies the equation

$$
D^{T} F_{M}^{ \pm}(.) \varphi=\varphi .
$$

Finally, the linear map

$$
F_{M}^{ \pm}: \Gamma_{0}^{\infty}\left(E^{*}\right) \ni \varphi \mapsto F_{M}^{ \pm}(.) \varphi \in \Gamma^{\infty}\left(E^{*}\right)
$$

is continuous.

\section{6. (Theorem)}

Let $(M, g)$ be a globally hyperbolic spacetime and $D \in \operatorname{Diffop}^{2}(E)$ a normally hyperbolic differential operator. Then the unique advanced and retarded Green functions $F_{\mathrm{M}}^{ \pm}(p)$ of $D$ at $p$ are of global order

$$
\text { ord } F_{\mathrm{M}}^{ \pm}(p) \leq 2 n+6 \text {. }
$$

More precisely, the linear map (23) extends to a continuous linear map

$$
F_{\mathrm{M}}^{ \pm}: \Gamma_{0}^{2(k++1)}\left(E^{*}\right) \ni \varphi \mapsto F_{\mathrm{M}}^{ \pm}(.) \varphi \in \Gamma^{k}\left(E^{*}\right)
$$

for all $\mathrm{k} \geq 2$ such that we still have

$$
D^{T} \quad F_{\mathrm{M}}^{ \pm}(.) \varphi=\varphi
$$

\subsection{Green Operator}

Let $(M, g)$ be a time-oriented Lorentz manifold and $D \in \operatorname{Diffop}^{2}(E)$ a normally hyperbolic differential operator. Then a continuous linear map

$$
G_{\cup}^{ \pm}: \Gamma_{0}^{\infty}(E) \rightarrow \Gamma^{\infty}(E)
$$

with
i.) $D G_{\mathrm{M}}^{ \pm}=i d \Gamma_{0}^{\infty}(E)$,
ii.) $\left.G_{\mathrm{M}}^{ \pm} D\right|_{\Gamma_{0}^{\infty}}=i d \Gamma_{0}^{\infty}(E)$,
iii) $\operatorname{Supp}\left(G_{\mathrm{M}}^{ \pm} u\right) \subseteq J_{\mathrm{M}}^{ \pm}(\operatorname{Supp} u)^{c 1}$ for all $u \in \Gamma_{0}^{\infty}(E)$.

is called an advanced and retarded Green operator for D respectively

\section{8. (Proposition) Green Operators and Fundamental Solutions}

Let $(M, g)$ be a time-oriented Lorentz manifold and $D \in \operatorname{Diffop}^{2}(E)$ a normally hyperbolic differential operator.

i.) Assume $\left\{G_{\mathrm{M}}^{ \pm}(p)\right\}$ is a family of global advanced or retarded fundamental solutions of $D^{T}$ at every point $P \in \mathrm{M}$ with the following property: for every test section $u \in \Gamma_{0}^{\infty}(E)$ the section $p \mapsto G_{\mathrm{M}}^{ \pm}(p) u$ is a smooth section of $E$ depending continuously on $u$ and satisfying $D G_{\mathrm{M}}^{ \pm}() u=$.$u .Then$

$$
\left(G_{\mathrm{M}}^{ \pm} u\right)(p)=G_{\mathrm{M}}^{\mp}(p) u
$$

yield advanced or retarded Green operator for $\mathrm{D}$, respectively.

ii.) Assume $G_{\mathrm{M}}^{ \pm}$are advanced or retarded Green operator for $D$, respectively. Then $G_{\mathrm{M}}^{ \pm}(p): \Gamma_{0}^{\infty}(E) \rightarrow C$ defined by

$$
\left(G_{\mathrm{M}}^{ \pm}\right)(p) u=\left(G_{\mathrm{M}}^{\mp} u\right)(p)
$$

defines a family of advanced and retarded fundamental solutions of $D^{T}$ at every point $P \in \mathrm{M}$ with the properties described in i.), respectively.

\section{9. (Proposition)}

Let $(M, g)$ be globally hyperbolic and let $D \in \operatorname{Diffop}^{2}(E)$ be a normally hyperbolic differential operator with advanced and retarded Green operators $G_{\mathrm{M}}^{ \pm}: \Gamma_{0}^{\infty}(E) \rightarrow \Gamma^{\infty}(E)$.

i.) The dual map $\left(G_{\mathrm{M}}^{ \pm}\right)^{\prime}: \Gamma_{0}^{-\infty}\left(E^{*}\right) \rightarrow \Gamma^{-\infty}\left(E^{*}\right)$ is weak ${ }^{*}$ continuous and satisfies

$$
D^{T}\left(G_{\mathrm{M}}^{ \pm}\right)^{\prime}(\varphi)=\varphi=\left(G_{\mathrm{M}}^{ \pm}\right)^{\prime} D^{T} \varphi
$$

for all generalized sections $\varphi \in \Gamma_{0}^{-\infty}\left(E^{*}\right)$ with compact support .

ii.) for generalized section $\varphi \in \Gamma_{0}^{-\infty}\left(E^{*}\right)$ with compact support we have

\subsection{0. (Lemma)}

$$
\operatorname{Supp}\left(G_{\mathrm{M}}^{ \pm}\right)^{\prime}(\varphi) \subseteq J_{\mathrm{M}}^{ \pm}(\operatorname{Supp} \varphi) .
$$

Let $(M, g)$ be globally hyperbolic and let $D \in \operatorname{Diffop}^{2}(E)$ be a normally hyperbolic differential operator with advanced and retarded Green operators $G_{\mathrm{M}}^{ \pm}$, Moreover, denote the corresponding Green operator of 
$D^{T} \in \operatorname{Diffop}^{2}\left(E^{*}\right)$

by $F_{\mathrm{M}}^{ \pm}$.Then we have for $\varphi \in \Gamma_{0}^{\infty}\left(E^{*}\right)$ and $u \in \Gamma_{0}^{\infty}(E)$

\subsection{1(Theorem)}

$$
\int_{M}\left(F_{\mathrm{M}}^{ \pm} \varphi\right) \cdot u \mu_{g}=\int_{M} \varphi\left(G_{\mathrm{M}}^{ \pm} u\right) \mu_{g} .
$$

Let $(M, g)$ be a globally hyperbolic and $D \in \operatorname{Diffop}^{2}(E)$ be normally hyperbolic differential operator. Denote the global advanced and retarded Green operator of $D$ by $G_{\mathrm{M}}^{ \pm}$and those of $D^{T}$ by $F_{\mathrm{M}}^{ \pm}$respectively .

i.) For the dual operators we have

$$
\begin{aligned}
& \left.\left(G_{\mathrm{M}}^{ \pm}\right)^{\prime}\right|_{\Gamma_{0}^{\infty}\left(E^{*}\right)}=F_{\mathrm{M}}^{ \pm} \\
& \left.\left(F_{\mathrm{M}}^{ \pm}\right)^{\prime}\right|_{\Gamma_{0}^{\infty}\left(E^{*}\right)}=G_{\mathrm{M}}^{ \pm}
\end{aligned}
$$

ii.) The duals of the Green operators restrict to maps,

$$
\begin{aligned}
\left(G_{\mathrm{M}}^{ \pm}\right)^{\prime} ; \Gamma_{0}^{\infty}\left(E^{*}\right) & \rightarrow \Gamma^{\infty}\left(E^{*}\right) \\
\left(F_{\mathrm{M}}^{ \pm}\right)^{\prime} ; \Gamma_{0}^{\infty}(E) & \rightarrow \Gamma^{\infty}(E)
\end{aligned}
$$

which are continuous with respect to the $\ell_{0}^{\infty}$-and $\ell^{\infty}$-topo $\log y$, respectively.

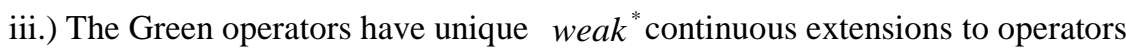

satisfying

$$
\begin{aligned}
G_{\mathrm{M}}^{ \pm} ; \Gamma_{0}^{-\infty}(E) & \rightarrow \Gamma^{-\infty}(E) \\
F_{\mathrm{M}}^{ \pm} ; \Gamma_{0}^{-\infty}\left(E^{*}\right) & \rightarrow \Gamma^{-\infty}\left(E^{*}\right)
\end{aligned}
$$

$$
\begin{aligned}
& \operatorname{Supp}\left(G_{\mathrm{M}}^{ \pm} u\right) \subseteq J_{\mathrm{M}}^{ \pm}(\operatorname{Suppu}) \\
& \operatorname{Supp}\left(F_{\mathrm{M}}^{ \pm} \varphi\right) \subseteq J_{\mathrm{M}}^{ \pm}(\operatorname{Supp} \varphi)
\end{aligned}
$$

respectively. for these extensions one has

$$
\begin{aligned}
& G_{\mathrm{M}}^{ \pm}=\left(\left.F_{\mathrm{M}}^{ \pm}\right|_{\Gamma_{0}^{\infty}\left(E^{*}\right)}\right)^{\prime} \\
& F_{\mathrm{M}}^{ \pm}=\left(\left.G_{\mathrm{M}}^{ \pm}\right|_{\Gamma_{0}^{\infty}\left(E^{*}\right)}\right)^{\prime}
\end{aligned}
$$

\subsection{2. (Theorem)}

Let $(M, g)$ be a globally hyperbolic spacetime and $D \in \operatorname{DiFFOp}^{2}(E)$ normally hyper-bolic with advanced and retarded Green operators $G_{\mathrm{M}}^{ \pm}$.

i.) The Green operators $G_{\mathrm{M}}^{ \pm} ; \Gamma_{0}^{-\infty}(E) \rightarrow \Gamma^{-\infty}(E)$, satisfy

$$
D G_{\mathrm{M}}^{ \pm}=i d_{\Gamma_{0}^{-\infty}(E)}=\left.G_{\mathrm{M}}^{ \pm} D\right|_{\Gamma_{0}^{-\infty}(E)}
$$

ii.) For every $v \in \Gamma_{0}^{-\infty}(E)$, every smooth spacelike Cauchy hypersurface $\iota: \Sigma \mapsto M$ with

$$
\operatorname{Supp} v \subseteq I_{\mathrm{M}}^{+}(\Sigma)
$$

and all $u_{0}, u_{0} \in \Gamma_{0}^{-\infty}\left(\imath^{\#} E\right)$, there exists a unique generalized section $u \in \Gamma^{-\infty}(E)$, with

$$
D u_{+}=v
$$

$$
\begin{gathered}
\text { Supp } u_{+} \subseteq J_{M}\left(\operatorname{Supp} u_{0} \cup \operatorname{Supp} \dot{u}_{0} \cup J_{M}^{+}(\operatorname{Supp} v)\right) \\
\text { Sing Supp } u_{+} \subseteq J_{M}^{+}(\operatorname{Supp} v) \\
\imath^{\#} u_{+}=u_{0} \text { and } \imath^{\#} \nabla_{n}^{E} u=u_{0} .
\end{gathered}
$$

The section $u_{+}$depends weak ${ }^{*}$ continuously on $v$ and continuouslyon $u_{0}, u_{0}$.

iii.) An analogous statement holds for the case $\operatorname{Supp} v \subseteq I_{M}^{-}(\Sigma)$.

\section{Conclusion}

The formulation of the Cauchy problem in Euclidean space with specified boundary condition is well known. In that formulation the traditional Green's function is involved in the construction of the solution. However one need a generalization of the Cauchy problem to spaces that are not Euclidean, such as Lorentzian manifolds, with pseudo-Riemannian metric .The consideration of this problem in such a geometrical Lorentzian manifold has very important impact on wave propagation with applications cosmic wave, Thus we have treated the formulation of Cauchy 
problem in Lorentzian manifolds. Here we also needed a generalizing form of Green's function . In order to find the inverse of Cauchy hyperbolic differential operator on a fiber bundle we also utilized the open mapping theorem appropriate to the problem. The solution appeared as a cross section of a fiber bundle ,that may be pulled down to base Lorentzian manifold to give the traditional local solution.

\section{References}

Baer, C., \& Strohmaier, A. (2015). An index theorem for Lorentzian manifolds with compact spacelike Cauchy boundary. arXiv preprint arXiv:1506.00959.

Bär, C., \& Fredenhagen, K. (2009). Quantum field theory on curved spacetimes: Concepts and mathematical foundations (Vol. 786): Springer.

Bär, C., \& Ginoux, N. (2012). Classical and quantum fields on Lorentzian manifolds. Global differential geometry (pp. 359-400): Springer.

Bar, C., Ginoux, N., \& Pfaffe, F. (2007). Wave equations on lorentzain manifold and quantization.ESL lectures in mathematics an physics Zurich: European Mathematic Society

Beem, J. K., Ehrlich, P., \& Easley, K. (1996). Global lorentzian geometry (Vol. 202): CRC Press.

Kreyszig, E. (1989). Introductory functional analysis with applications (Vol. 81): wiley New York.

Minguzzi, E., \& Sánchez, M. (2008). The causal hierarchy of spacetimes. Recent developments in pseudo-Riemannian geometry, ESI Lect. Math. Phys, 299-358.

Mühlhoff, R. (2011). Cauchy problem and Green's functions for first order differential operators and algebraic quantization. Journal of Mathematical Physics, 52(2), 022303.

O'neill, B. (1983). Semi-Riemannian Geometry With Applications to Relativity, 103 (Vol. 103): Academic press.

Stakgold, I., \& Holst, M. J. (2011). Green's functions and boundary value problems (Vol. 99): John Wiley \& Sons.

Waldmann, S. (2012). Geometric wave equations. arXiv preprint arXiv:1208.4706.

\section{Copyrights}

Copyright for this article is retained by the author(s), with first publication rights granted to the journal.

This is an open-access article distributed under the terms and conditions of the Creative Commons Attribution license (http://creativecommons.org/licenses/by/3.0/). 